\title{
DETECTION OF PHYTOCHEMICAL CONSTITUENT IN FLOWERS OF VIOLA ODORATA BY GAS CHROMATOGRAPHY-MASS SPECTROMETRY
}

\author{
SHAIMAA FAKHRI JASIM, NOOR NIHAD BAQER*, ESAM ABD ALRAHEEM
}

Ministry of Science and Technology, Directorate of Water and Environment. *Email: noornihadbaqer@gmail.com

Received: 15 December 2017, Revised and Accepted: 30 January 2018

\section{ABSTRACT}

Objective: Viola odorata has a characteristic as antifungal, antibacterial, anticancer, antioxidant, antiasthmatic, anti-inflammatory, anti-HIV, and antipyretic agents. The aim of this study was detected about bioactive compounds in the methanolic extract of $V$. odorata.

Methods: The methanolic extract was analyzed through gas chromatography-mass spectrometry (GC-MS) for the identification of different compounds.

Results: The current study investigated about phytochemicals in flowers of $V$. odorata. GC-MS analysis of the methanol extract of flowers showed 84 compounds. The highest concentration was for components which include ethanol, 2-(9,12-octadecadienyl oxy) -, (Z,Z)-; pentadecanoic acid; 1-pentacosanol; 1-pentacosanol; 2-furan carboxaldehyde, 5-(hydroxymethyl)-; 1,2 benzenedicarboxylic acid, diisooctyl ester; and docosane, 11-butyl- and gamma-sitosterol. The peak area and retention time for each components, respectively, were (15.709, 25.51\%), (14.015, 19.51\%), (29.914, 4.69\%), (27.292, 3.95\%), (5.707, 4.05\%), (20.357, 3.91\%), (18.289, 2.48\%), and (30.431, 2.37\%). While the others components ranged the peak area from $2.03 \%$ to $0.05 \%$.

Conclusions: These results indicate that the flowers of $V$. odorata contain the numerous components which have medical importance and this study was one of the first studies to detect phytochemicals in V. odorata.

Keywords: Viola odorata, Gas chromatography-mass spectrometry analysis, Phytochemicals, Pentadecanoic acid, Gamma-sitosterol.

(C) 2018 The Authors. Published by Innovare Academic Sciences Pvt Ltd. This is an open access article under the CC BY license (http://creativecommons. org/licenses/by/4. 0/) DOI: http://dx.doi.org/10.22159/ajpcr.2018.v11i5.24288

\section{INTRODUCTION}

The medicinal herbs obtained much importance in recent years due to extensive applications of its bioactive molecules. The different strategies have been advanced for the selection of specific herbs for the study. The herbs selected were screened for the active phytoconstituents. The specific component present in the herbs was active subjected to isolation with different analytical techniques. The analogs of isolated molecules are characterized, and structural modification has been done to enhance the desired activity and minimize the unfavorable side effects [1]. The Violaceae is a family which contains about 900 species belonging to 22 genera; this family is a medium-sized of perennial or rarely annual herbs or shrubs, including the violets or pansies. It is cosmopolitan, but more typical of the temperate regions and tends to be restricted to higher mountainous areas. The Viola L. is the largest genus of the family and has about 400 species in the world [2].

Viola odorata perennial herb, rhizome short, thick leaves are heartshaped, slightly downy, especially beneath, on stalks rising alternately from a creeping rhizome or underground stem. The flowers are generally deep purple, giving their name to the color which was called for it, pale rose-colored or white variations are also frequent, and all these tints may sometimes be discovered in different plants growing on the same land. The flowers are full of honey and are constructed for bee visitors, but bloom before it is really bee time, so that it is rare that a violet flower is found setting seed [3] V. odorata has a characteristic as antifungal, antibacterial, anticancer, antioxidant, antiasthmatic, antiinflammatory, anti-HIV, and antipyretic agents [4]. The flowers infusion used for jaundice, eczema, febrifuge, antiallergic, blood cleansing, and migraine [5,6]. So Shafi and Tabassum [7] revealed the importance of herbal remedies, such as of fruits of Eriobotrya japonica has beneficial effects on serum glucose levels. While Gulilat et al. [8] showed the importance of gas chromatography-mass spectrometry (GC-MS) in the phytochemical analysis which revealed the presence of various secondary metabolites, namely, flavonoids, alkaloids, terpenoids, saponins, and carotenoid in rue extract, it has a property antimicrobial agents in drugs development of infectious disease. This study aimed to detect about bioactive compounds in the methanolic extract of $V$. odorata.

\section{METHODS}

\section{Plant material}

$V$. odorata L. plant which authenticated by the National Herbarium of Iraq Botany Directorate at Abu-Ghraib was collected in April and July. The flowers dried at room temperature $25^{\circ} \mathrm{C}$ in the shade for $(10)$ days then it was crushed into powder by electric Grinder and weighted.

\section{Preparation of extracts}

$100 \mathrm{~g}$ of crushed powder (flowers) of $V$. odorata L. Plant was macerated for $36 \mathrm{~h}$ with shaking at room temperature $25^{\circ} \mathrm{C}$ in $1 \mathrm{~L}$ methanol, and the resulting extract was filtered. The residue was re-extracted twice for complete exhaustion. The obtained filtrates were combined and concentrated using a rotary evaporator to get the dry extract. The dried extract was dissolved in methanol and stored at $4^{\circ} \mathrm{C}$ in a refrigerator [9].

GC-MS analysis

The methanolic extract was analyzed through GC-MS for the identification of different compounds.

Instruments and chromatographic conditions GC Program Column: Elite-5MS (5\% diphenyl/95\% dimethyl polysiloxane), $30 \times$ $0.25 \mathrm{~mm} \times 0.25 \mathrm{~m}$ df. Equipment: GC Shimadzu ap 2010 plus. Carrier gas: $3 \mathrm{ml} / \mathrm{min}$, Split: 10:1. Detector: Mass detector Quader mass goldPerkin Elmer

Software: Turbomass 5.2. Sample injected: $2 \mu \mathrm{l}$ 
Oven temperature program

- $80^{\circ} \mathrm{C}-2$ min hold. Up to $300^{\circ} \mathrm{C} 120 \mathrm{~min}$ at the rate of $10^{\circ} \mathrm{C} / \mathrm{min}$.

- Injector temperature $280^{\circ} \mathrm{C}$. Total GC is running time $30 \mathrm{~min}$.

\section{MS program}

- Library used NIST 10 Version-year 2010. Inlet line temperature $280^{\circ} \mathrm{C}$.

- Source temperature $200^{\circ} \mathrm{C}$. Electron energy: $70 \mathrm{eV}$. Mass scan (m/z): 40-600.

- Solvent delay: 0-2 min. Total MS running time: $30 \mathrm{~min}$

- $\quad$ The extract was dissolved in methanol and filtered with Elite- 5 MS column and analyzed in GC-MS for different constituents. The phytoconstituents obtained as a result was interpreted on mass spectrum GCMC using NIST (2010).

\section{RESULT}

GC-MS of the methanol extract of $V$. odorata flowers showed 84 peaks. The constituents were shown in Fig. 1 and Table 1 with their retention time (RT), molecular formula, molecular weight, concentration (peak area \%), and chemical structures. GC-MS analysis showed 84 compounds the highest concentration was for components which include ethanol, 2-(9,12-octadecadienyloxy)-, (Z,Z)-; pentadecanoic acid; 1-pentacosanol; 1-pentacosanol; 2-furancarboxaldehyde, 5-(hydroxymethyl)-; 1,2 benzenedicarboxylic acid, diisooctyl ester; docosane, and 11-butyl- and gamma-sitosterol. The RT and peak area for each components, respectively, were (15.709, 25.51\%), (14.015, $19.51 \%),(29.914,4.69 \%),(27.292,3.95 \%),(5.707,4.05 \%),(20.357$, $3.91 \%),(18.289,2.48 \%)$, and $(30.431,2.37 \%)$. While the remain of the components ranged the peak area from $2.03 \%$ to $0.05 \%$.

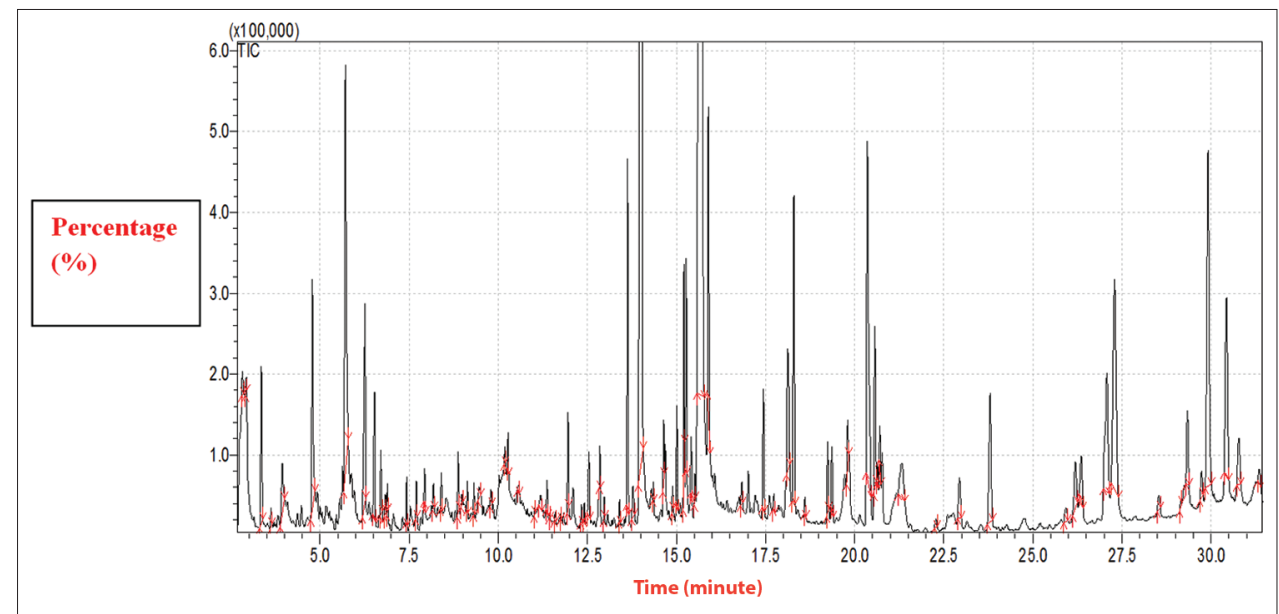

Fig. 1: Gas chromatography-mass spectrometry chromatogram of methanol extract of Viola odorata

Table 1: The compounds identified from methanol extract of $V$. odorata flowers by gas chromatography-mass spectrometry

\begin{tabular}{|c|c|c|c|c|c|}
\hline NO & RT & Compound & Molecular formula & MW & Peak \\
\hline 1 & 2.593 & 3-Buten-2-one, 4-(dimethylamino)-4-(1-piperidinyl) & $\mathrm{C}_{11} \mathrm{H}_{20} \mathrm{~N}_{2} \mathrm{O}$ & 196 & 0.05 \\
\hline 2 & 2.820 & Propanoic acid, 2-(aminooxy)- & $\mathrm{C}_{3} \mathrm{H}_{7} \mathrm{NO}_{3}$ & 105 & 0.13 \\
\hline 3 & 2.930 & Glycerin & $\mathrm{C}_{3} \mathrm{H}_{8} \mathrm{O}_{3}$ & 92 & 0.06 \\
\hline 4 & 3.347 & 1-Decyne & $\mathrm{C}_{10} \mathrm{H}_{18}$ & 138 & 1.23 \\
\hline 5 & 3.624 & Benzene acetaldehyde & $\mathrm{C}_{8} \mathrm{H}_{8} \mathrm{O}$ & 120 & 0.11 \\
\hline
\end{tabular}<smiles>CCCCC(O)COC(O[AlH2])C(=O)O</smiles> 
Table 1: (Continued)

\begin{tabular}{|c|c|c|c|c|c|c|}
\hline NO & RT & Compound & Molecular formula & MW & Peak area & Chemical structure \\
\hline 6 & 3.938 & $\begin{array}{l}\text { Benzaldehyde, } \\
\text { 3-phenoxy-, (4,6-dimethyl-1,3,5-triazin-2-yl) hydrazone }\end{array}$ & $\mathrm{C}_{16} \mathrm{H}_{15} \mathrm{~N}_{7} \mathrm{O}$ & 321 & 0.51 & \\
\hline 7 & 4.784 & 4H-Pyran-4-one, 2,3-dihydro-3,5-dihydroxy-6-methyl- & $\mathrm{C}_{6} \mathrm{H}_{8} \mathrm{O}_{4}$ & 144 & 1.83 & \\
\hline 8 & 5.707 & 2-Furancarboxaldehyde, 5-(hydroxymethyl)- & $\mathrm{C}_{6} \mathrm{H}_{6} \mathrm{O}_{3}$ & 126 & 4.05 & \\
\hline 9 & 6.258 & Nonanoic acid & $\mathrm{C}_{9} \mathrm{H}_{18} \mathrm{O}_{2}$ & 158 & 1.75 & \\
\hline 10 & 6.522 & 8-Nonynoic acid & $\mathrm{C}_{9} \mathrm{H}_{14} \mathrm{O}_{2}$ & 154 & 0.90 & \\
\hline 11 & 6.703 & Salicylic acid & $\mathrm{C}_{7} \mathrm{H}_{6} \mathrm{O}_{3}$ & 138 & 0.14 & \\
\hline 12 & 6.829 & Methyl 2,3-anhydro-.beta.-d-ribofuranoside & $\mathrm{C}_{6} \mathrm{H}_{10} \mathrm{O}_{4}$ & 146 & 0.11 & \\
\hline 13 & 6.890 & 2-Methoxy-4-vinylphenol & $\mathrm{C}_{9} \mathrm{H}_{10} \mathrm{O}_{2}$ & 150 & 0.13 & \\
\hline 14 & 7.425 & Eugenol & $\mathrm{C}_{10} \mathrm{H}_{12} \mathrm{O}_{2}$ & 164 & 0.22 & \\
\hline 15 & 7.703 & 8-Methyl-6-nonenoic acid & $\mathrm{C}_{10} \mathrm{H}_{18} \mathrm{O}_{2}$ & 170 & 0.19 & \\
\hline 16 & 7.933 & Decane, 2-methyl- & $\mathrm{C}_{11} \mathrm{H}_{24}$ & 156 & 0.19 & \\
\hline 17 & 8.181 & Benzeneethanol, 4-hydroxy- & $\mathrm{C}_{8} \mathrm{H}_{10} \mathrm{O}_{2}$ & 138 & 0.15 & \\
\hline
\end{tabular}


Table 1: (Continued)

\begin{tabular}{|c|c|c|c|c|c|c|}
\hline No & RT & Compound & Molecular formula & MW & Peak area & Chemical structure \\
\hline 18 & 8.399 & Benzaldehyde, 2-hydroxy-6-methyl- & $\mathrm{C}_{8} \mathrm{H}_{8} \mathrm{O}_{2}$ & 136 & 0.22 & \\
\hline 19 & 8.873 & Suberic acid monomethyl ester & $\mathrm{C}_{9} \mathrm{H}_{16} \mathrm{O}_{4}$ & 188 & 0.33 & \\
\hline 20 & 8.979 & 4-(2-Methoxyethyl)-2-methylphenol & $\mathrm{C}_{10} \mathrm{H}_{14} \mathrm{O}_{2}$ & 166 & 0.07 & \\
\hline 21 & 9.129 & Octane, 2,3,3-trimethyl- & $\mathrm{C}_{11} \mathrm{H}_{24}$ & 156 & 0.14 & \\
\hline 22 & 9.317 & Cyclooctane & $\mathrm{C}_{8} \mathrm{H}_{16}$ & 122 & 0.16 & \\
\hline 23 & 9.467 & Octanedioic acid & $\mathrm{C}_{8} \mathrm{H}_{14} \mathrm{O}_{4}$ & 174 & 0.11 & \\
\hline 24 & 9.789 & 3-Hydroxy-4-methoxybenzoic acid & $\mathrm{C}_{8} \mathrm{H}_{8} \mathrm{O}_{4}$ & 168 & 0.11 & \\
\hline 25 & 10.184 & Didodecyl phthalate & $\mathrm{C}_{32} \mathrm{H}_{54} \mathrm{O}_{4}$ & 502 & 0.06 & \\
\hline 26 & 10.273 & Dodecane, 2,6,11-trimethyl- & $\mathrm{C}_{15} \mathrm{H}_{32}$ & 212 & 0.17 & \\
\hline 27 & 10.561 & Azelaic acid & $\mathrm{C}_{9} \mathrm{H}_{16} \mathrm{O}_{4}$ & 188 & 0.05 & \\
\hline 28 & 11.019 & 3,3-Dimethylacryloyl chloride & $\mathrm{C} 5 \mathrm{H} 7 \mathrm{ClO}$ & 118 & 0.03 & \\
\hline 29 & 11.188 & Epi-inositol & $\mathrm{C}_{6} \mathrm{H}_{12} \mathrm{O}_{6}$ & 180 & 0.12 & \\
\hline 30 & 11.365 & 2-Bromo dodecane & $\mathrm{C}_{12} \mathrm{H}_{25} \mathrm{Br}$ & 248 & 0.15 & \\
\hline
\end{tabular}


Table 1: (Continued)

\begin{tabular}{|c|c|c|c|c|c|c|}
\hline NO & RT & Compound & Molecular formula & MW & Peak area & Chemical structure \\
\hline 31 & 11.445 & 2-Benzoyl-3,4-acetone-d-galactosan & $\mathrm{C}_{16} \mathrm{H}_{18} \mathrm{O}_{6}$ & 306 & 0.02 & \\
\hline 32 & 11.595 & dl-Serine & $\mathrm{C}_{3} \mathrm{H}_{7} \mathrm{NO}_{3}$ & 105 & 0.05 & \\
\hline 33 & 11.758 & 2,5-Dihydroxy-4-isopropyl-2,4,6-cycloheptatrien-1-one & $\mathrm{C}_{10} \mathrm{H}_{12} \mathrm{O}_{3}$ & 180 & 0.03 & \\
\hline 34 & 11.958 & Tetradecanoic acid & $\mathrm{C}_{14} \mathrm{H}_{28} \mathrm{O}_{2}$ & 228 & 0.48 & \\
\hline 35 & 12.334 & Undecyl trifluoroacetate & $\mathrm{C}_{13} \mathrm{H}_{23} \mathrm{~F}_{3} \mathrm{O}_{2}$ & 268 & 0.09 & \\
\hline 36 & 12.407 & 2-Bromo dodecane & $\mathrm{C}_{12} \mathrm{H}_{25} \mathrm{Br}$ & 248 & 0.09 & \\
\hline 37 & 12.540 & 3-Hydroxy-4,5-dimethoxybenzoic acid & $\mathrm{C}_{9} \mathrm{H}_{10} \mathrm{O}_{5}$ & 198 & 0.49 & \\
\hline 38 & 12.581 & 2-Pentadecanone, 6,10,14-trimethyl- & $\mathrm{C}_{18} \mathrm{H}_{36} \mathrm{O}$ & 268 & 0.16 & \\
\hline 39 & 12.973 & Pentadecanoic acid & $\mathrm{C}_{15} \mathrm{H}_{30} \mathrm{O}_{2}$ & 242 & 0.10 & \\
\hline 40 & 13.403 & Undecane, 3,8-dimethyl- & C13H28 & 184 & 0.13 & \\
\hline 41 & 13.626 & Hexadecanoic acid, methyl ester & $\mathrm{C}_{17} \mathrm{H}_{34} \mathrm{O}_{2}$ & 270 & 2.00 & \\
\hline 42 & 13.776 & 2-Tridecenal, (E)- & $\mathrm{C}_{13} \mathrm{H}_{24} \mathrm{O}$ & 196 & 0.26 & \\
\hline 43 & 14.015 & Pentadecanoic acid & $\mathrm{C}_{15} \mathrm{H}_{30} \mathrm{O}_{2}$ & 242 & 19.51 & \\
\hline 44 & 14.352 & $\begin{array}{l}\text { Card-20 (22)-enolide, 3-[(2,6-dideoxy-4-0-. } \\
\text { beta.-D-glucopyranosyl-3-0-methyl-. } \\
\text { beta.-D-ribo-hexopyranosyl) oxy]-5,14-dihydroxy-19-oxo }\end{array}$ & $\mathrm{C}_{36} \mathrm{H}_{54} \mathrm{O}_{14}$ & 710 & 0.07 & \\
\hline 45 & 14.637 & 7-Methyl-Z-tetradecen-1-ol acetate & $\mathrm{C}_{17} \mathrm{H}_{32} \mathrm{O}_{2}$ & 268 & 0.31 & \\
\hline 46 & 14.889 & Eicosanoic acid & $\mathrm{C}_{20} \mathrm{H}_{40} \mathrm{O}_{2}$ & 312 & 0.14 & \\
\hline 47 & 15.011 & Oxacycloheptadec-8-en-2-one & $\mathrm{C}_{16} \mathrm{H}_{28} \mathrm{O}_{2}$ & 252 & 0.62 & \\
\hline 48 & 15.213 & 9,12-Octadecadienoic acid (Z, Z)-, methyl ester & $\mathrm{C}_{19} \mathrm{H}_{34} \mathrm{O}_{2}$ & 294 & 1.12 & \\
\hline 49 & 15.274 & $\begin{array}{l}\text { 9,12,15-Octadecatrienoic acid, 2,3-dihydroxypropyl } \\
\text { ester, (Z, Z, Z)- }\end{array}$ & $\mathrm{C}_{21} \mathrm{H}_{36} \mathrm{O}_{4}$ & 352 & 1.11 & \\
\hline 50 & 15.414 & Phytol & $\mathrm{C}_{20} \mathrm{H}_{40} \mathrm{O}$ & 296 & 0.32 & \\
\hline
\end{tabular}


Table 1: (Continued)

\begin{tabular}{|c|c|c|c|c|c|c|}
\hline NO & RT & Compound & Molecular formula & MW & Peak area & Chemical structure \\
\hline 51 & 15.513 & Octadecanoic acid, methyl ester & $\mathrm{C}_{19} \mathrm{H}_{38} \mathrm{O}_{2}$ & 298 & 0.14 & \\
\hline 52 & 15.709 & Ethanol, 2-(9,12-octadecadienyloxy)-, (Z, Z)- & $\mathrm{C}_{20} \mathrm{H}_{38} \mathrm{O}_{2}$ & 310 & 25.51 & \\
\hline 53 & 15.896 & Octadecanoic acid & $\mathrm{C}_{18} \mathrm{H}_{36} \mathrm{O}_{2}$ & 284 & 2.05 & \\
\hline 54 & 16.829 & Oleyl alcohol, trifluoroacetate & $\mathrm{C}_{20} \mathrm{H}_{35} \mathrm{~F}_{3} \mathrm{O}_{2}$ & 364 & 0.15 & \\
\hline 55 & 17.436 & Eicosane & $\mathrm{C}_{20} \mathrm{H}_{42}$ & 282 & 0.88 & \\
\hline 56 & 17.734 & Cyclopentaneundecanoic acid, methyl ester & $\mathrm{C}_{17}^{20} \mathrm{H}_{32} \mathrm{O}_{2}$ & 268 & 0.12 & \\
\hline 57 & 18.119 & Deoxyspergualin & $\mathrm{C}_{17} \mathrm{H}_{37} \mathrm{~N}_{7} \mathrm{O}_{3}$ & 387 & 1.28 & \\
\hline 58 & 18.289 & Docosane, 11-butyl- & $\mathrm{C}_{26} \mathrm{H}_{54}$ & 366 & 2.48 & \\
\hline 59 & 18.604 & Sulfurous acid, 2-propyl tridecyl ester & $\mathrm{C}_{16} \mathrm{H}_{34} \mathrm{O}_{3} \mathrm{~S}$ & 306 & 0.12 & \\
\hline 60 & 19.244 & 1,2-Propanediol, 3-benzyloxy-1,2-diacetyl- & $\mathrm{C}_{14} \mathrm{H}_{18} \mathrm{O}_{5}$ & 266 & 0.48 & \\
\hline 61 & 19.360 & Tetratetracontane & $\mathrm{C}_{44} \mathrm{H}_{90}$ & 618 & 0.50 & \\
\hline 62 & 19.804 & Undecane, 2-methyl- & $\mathrm{C}_{12}^{44} \mathrm{H}_{26}^{90}$ & 170 & 0.36 & \\
\hline 63 & 20.357 & 1,2-Benzenedicarboxylic acid, diisooctyl ester & $\mathrm{C}_{24} \mathrm{H}_{38} \mathrm{O}_{4}$ & 390 & 3.91 & \\
\hline 64 & 20.564 & $\begin{array}{l}\text { (2,3-Diphenylcyclopropyl) methyl phenyl sulfoxide, } \\
\text { trans- }\end{array}$ & $\mathrm{C}_{22} \mathrm{H}_{20} \mathrm{OS}$ & 332 & 1.26 & \\
\hline 65 & 20.635 & $\begin{array}{l}\text { (2,3-Diphenylcyclopropyl) methyl phenyl sulfoxide, } \\
\text { trans- }\end{array}$ & $\mathrm{C}_{22} \mathrm{H}_{20} \mathrm{OS}$ & 332 & 0.09 & \\
\hline 66 & 20.704 & Pentacosane, 13-undecyl- & $\mathrm{C}_{36} \mathrm{H}_{74}$ & 506 & 0.23 & \\
\hline 67 & 21.325 & Acetic acid n-octadecyl ester & $\mathrm{C}_{20} \mathrm{H}_{40} \mathrm{O}_{2}$ & 312 & 0.74 & \\
\hline 68 & 22.281 & Benzene, (3-ethenyl-5,5-dimethylhexyl)- & $\mathrm{C}_{16} \mathrm{H}_{24}$ & 216 & 0.09 & \\
\hline
\end{tabular}


Table 1: (Continued)

\begin{tabular}{|c|c|c|c|c|c|c|}
\hline NO & RT & Compound & Molecular formula & MW & Peak area & Chemical structure \\
\hline 69 & 22.939 & Oxalic acid, allyl tetradecyl ester & $\mathrm{C}_{19} \mathrm{H}_{34} \mathrm{O}_{4}$ & 326 & 0.49 & \\
\hline 70 & 23.799 & 1-Octacosanol & $\mathrm{C}_{28} \mathrm{H}_{58} \mathrm{O}$ & 410 & 1.69 & \\
\hline 71 & 25.931 & Ethanol, 2-(3,3-dimethylbicyclo[2.2.1]hept-2-ylidene)- & $\mathrm{C}_{11}^{28} \mathrm{H}_{18}^{38} \mathrm{O}$ & 166 & 0.19 & \\
\hline 72 & 26.193 & Imidazole, 4-fluoro-5-aminocarbonyl- & $\mathrm{C}_{4} \mathrm{H}_{4} \mathrm{FN}_{3} \mathrm{O}$ & 129 & 0.63 & \\
\hline 73 & 26.353 & 1,7-Dimethyl-4-(1-methylethyl) cyclodecane & $\mathrm{C}_{15} \mathrm{H}_{30}$ & 210 & 0.50 & \\
\hline 74 & 27.078 & Heptacosyl heptafluorobutyrate & $\mathrm{C}_{31} \mathrm{H}_{55} \mathrm{~F}_{7} \mathrm{O}_{2}$ & 592 & 2.03 & \\
\hline 75 & 27.292 & 1-Pentacosanol & $\mathrm{C}_{25} \mathrm{H}_{52} \mathrm{O}$ & 368 & 3.95 & \\
\hline 76 & 28.530 & 1-Heptatriacotanol & $\mathrm{C}_{37}^{25} \mathrm{H}_{76}^{52} \mathrm{O}$ & 536 & 0.15 & \\
\hline 77 & 29.154 & Silane, dimethyl (2-nitrophenoxy) tetradecyloxy- & $\mathrm{C}_{22}^{31} \mathrm{H}_{39} \mathrm{NO}_{4} \mathrm{Si}$ & 409 & 0.05 & \\
\hline 78 & 29.339 & 3-Eicosene, (E)- & $\mathrm{C}_{20} \mathrm{H}_{40}$ & 280 & 0.69 & \\
\hline 79 & 29.730 & 22,23-Dibromostigmasterol acetate & $\mathrm{C}_{31} \mathrm{H}_{50} \mathrm{Br}_{2} \mathrm{O}_{2}$ & 612 & 0.26 & \\
\hline 80 & 29.914 & Triacontyl pentafluoropropionate & $\mathrm{C}_{33} \mathrm{H}_{6} 1 \mathrm{~F}_{5} \mathrm{O}_{2}$ & 584 & 4.69 & \\
\hline 81 & 30.431 & Gamma.-Sitosterol & $\mathrm{C}_{29} \mathrm{H}_{50} \mathrm{O}$ & 414 & 2.37 & \\
\hline 82 & 30.871 & Cholest-5-en-3-ol, 24-propylidene-, (3.beta.)- & $\mathrm{C}_{30} \mathrm{H}_{50} \mathrm{O}$ & 426 & 0.63 & \\
\hline 83 & 31.348 & 3-Hydroxyspirost-8-en-11-one & $\mathrm{C}_{27} \mathrm{H}_{40} \mathrm{O}_{4}$ & 428 & 0.15 & \\
\hline 84 & 31.883 & Ergost-5,8 (14)-dien-3-ol & $\mathrm{C}_{28} \mathrm{H}_{46} \mathrm{O}$ & 398 & 0.28 & \\
\hline
\end{tabular}

V. odorata: Viola odorata, MW: Molecular weight, RT: Retention time

\section{DISCUSSION}

GC analysis was not detected for the flower of V. odorata previously, while Hammami et al. [10] showed of the active components in volatile oils in Viola odorata by using GC, it revealed of the presence 63 identified volatile constituents, the main components were including: 1-phenyl butanone (22.43\%), linalool (7.33\% \%),benzyl alcohol (5.65\%), $\alpha$-cadinol (4.91), globulol $(4.32 \%)$ and viridiflorol (3.51\%). Pulegone(3.33\%), epi- $\alpha-$ cadinol (3.05\%), terpinen-4-ol (2.31\%), germacrene A (1.99\%) and paramethyl anisole $(1.09 \%)$ were found to be the main compounds [11] showed in GC analysis of Hybanthus enneaspermus which belongs to the family Violaceae, it contains the major phytoconstituents were (5E,13E)-5,13-Doco sadienoic acid (20.90\%) and Cedran-diol, 8S,
14- (13.02). The results shown that the flower $V$. odorata contains active compounds that have medical importance such as eugenol has the characteristic as anti-tumor necrosis factor, antioxidant, antiprostaglandin, antipyretic, antiradicular, anti-salmonella, antiseptic, antiseptic, fungicide, antiestrogenic, antigenotoxic and antiviral [12], and anti-tumor [13]. While gamma-sitosterol used as anti-diabetic, anti-angiogenic, anticancer, antimicrobial, anti-inflammatory, antidiarrheal, and antiviral [14]. Phytol has the property as antimicrobial, anti-inflammatory, antioxidant, diuretic, antimicrobial, anticancer, anti-inflammatory, anti-diuretic, immunostimulatory and anti-diabetic, and antimycobacterial activity [15]. While tetradecanoic acid used as antioxidant, lubricant, hypercholesterolemic, cancer-preventive, and cosmetic. Whereas hexadecanoic acid, methyl ester has the 
ability as antioxidant, flavor, antifibrinolytic, hypocholesterolemic, anti-androgenic, lubricant, hemolytic, 5-alpha reductase inhibitor, nematicide, and anti-alopecic [16]. As for the compound octacosanol used as anticancer, cholesterol-lowering effect, anticoagulant, increase stamina and improve strength and reaction time for athletes [17]. Octadecanoic acid, methyl ester has the property as antioxidant, antibacterial, antifungal, anti-inflammatory, antiarthritic, antihistimic, anti-coronary, hypocholesterolemic, anticancer, hepatoprotective action, soap, lumbricant, and cosmetics [18].

\section{CONCLUSION}

This study showed through the GC analysis, the flowers of $V$. odorata contains many active compounds which have the medical importance and bioactivity. These compounds can be isolated and tested for the cellular toxicity to determine the safety of their usage in the treatment of diseases.

\section{CONFLICTS OF INTEREST}

The authors have not declared any conflict of interest. But have a contribution to some research in Ministry of science and technology \ Directorate of Water and Environment $\backslash$ Iraq.

\section{REFERENCES}

1. Patil SJ, Venkatesh S, Vishwanatha T, Banagar SR, Banaga RJ, Patil SB. GCMS analysis of bioactive constituents from the petroleum ether extract of Citrus medica Seeds. World J Pharm Pharm Sci 2014;3:1239-49.

2. Bağc1 Y, Dinç M, Öztürk M. Morphological, anatomical and ecological study on turkish endemic viola yildirimlii $\mathrm{m}$. dinç \& y. bağcı. Int J Nat Eng Sci 2008;2:1-5.

3. Alwash BM. Phytochemical and Cytotoxic Studies of Viola odorata L. Cultivated in Iraq. Ministry of Higher Education, Scientific Research, University of Baghdad, Collage Science for Women. Thesis; 2006.

4. Barekat T, Otroshy M, Zadeh BS, Sadr-arhami A, Mokhtari A. A novel approach for breaking seed dormancy and germination in Viola odorata (A medicinal plant). J Novel Appl Sci 2013;2:513-6.

5. Amiri MS, Joharchi MR, Yazdi ME. Ethno-medicinal plants used to cure jaundice by traditional healers of mashhad, Iran. Iran J Pharm Res 2014;13:157-62.
6. Ahvazi A, Sigaroodi FK, Charkhchiyan MM, Mojab F, Mozaffarian V, Zakeri H. Introduction of medicinal plants species with the most traditional usage in alamut region. Iran $\mathrm{J}$ Pharm Res 2012;11:185-94.

7. Shafi S, Tabassum N. Phytochemical screening and renal effects of ethanolic extract of Eriobotrya japonica fruits and seed in alloxan induced diabetic rats. Int J Curr Pharm Res 2018;10:3-7.

8. Gulilat H, Work A, Sahabjada, Jafri A, Arshad MD, Malik T. The phytochemical investigation, GC-MS profile and antimicrobial activity of a medicinal plant Ruta graveolens L. from Ethiopia. Int J Pharm Pharm Sci 2017;9:29-34.

9. Mittal S. Thin layer chromatography and high presure liquid chromatography profiling of plant extracts of Viola odorata Linn. Int J Pharm Biosci 2013;4:542-9.

10. Hammami I, Kamoun N, Rebai A. Biocontrol of Botrytis cinerea with essential oil and methanol extract of Viola odorata L. flowers. Arch Appl Sci Res 2011;3:44-51.

11. Anand T, Gokulakrishnan K. Phytochemical analysis of Hybanthus enneaspermus using UV, FTIR and GC- MS. IOSR J Pharm 2012;2:520-4.

12. Duke JA. Handbook of Phytochemical Constituents of GRAS Herbs and other Economic Plants. Boca Raton, FL: CRC Press; 1992.

13. Zheng GQ, Kenney PM, Lam LK. Sesquiterpenes from clove (Eugenia caryophyllata) as potential anticarcinogenic agents. J Nat Prod 1992;55:999-1003.

14. Karthikeyan M, Balasubramanian T, Kumar P. Phytochemical screening of ethanolic extract of Premna coriacea through an integrative GC-MS and LC-MS. Asian J Pharm Educ Res 2017;6:44-55.

15. Rajesh KD, Vasantha S, Panneerselvam A, Rajesh NV, Jeyathilakan N. Phytochemical analysis, In vitro antioxidant potential and Gas Chromatography-mass spectrometry studies of Dicranopteris linearis. Asian J Pharm Clin Res 2016;9:220-5.

16. Markkas N, Govindharajalu M. Determination of phytocomponents in the methanolic of Mollugo cerviana by GC-MS analysis. Int J Res Biol Sci 2015;5:26-9.

17. Raman BV, Samul LA, Saradhi MP, Rao BN, Vamsi Krishna AN, Sudhakar M, Radhakrishnan TM. Antibacterial, antioxidant activity and GC-MS analysis of Eupatorium odoratum. Asian J Pharm Clin Res 2012;5:99-106.

18. Elangovan M, Dhanarajan MS, Elangovan I. Determination of bioactivitie compounds from the petroleum ether extract of Moringa oleifera and Phyllanthus emblica using GC-MS analysis. World J Pharm Res 2015;4:1284-98. 